\title{
Artificial Intelligence Architecture Based on Planar LiDAR Scan Data to Detect Energy Pylon Structures in a UAV Autonomous Detailed Inspection Process
}

\author{
Matheus F. Ferraz ${ }^{1(\otimes)}\left(\mathbb{D}\right.$, Luciano B. Júnior ${ }^{1}$, Aroldo S. K. Komori ${ }^{1}$, \\ Lucas C. Rech ${ }^{1}$, Guilherme H. T. Schneider ${ }^{1}$, Guido S. Berger ${ }^{1}$ (D), \\ Álvaro R. Cantieri ${ }^{2}$ (D) José Lima ${ }^{3}$, and Marco A. Wehrmeister ${ }^{1}$ (D) \\ 1 The Federal University of Technology - Paraná, Curitiba, Brazil \\ \{mferraz, lucjun, aroldok, lucasrech, ghideki\}@alunos.utfpr.edu.br, \\ wehrmeister@utfpr.edu.br \\ ${ }^{2}$ Federal Institute of Paraná, Curitiba, Brazil \\ alvaro.cantieri@ifpr.edu.br \\ 3 Research Centre in Digitalization and Intelligent Robotics (CeDRI), \\ Instituto Politécnico de Bragança, Portugal and INESC TEC, Porto, Portugal \\ jllima@ipb.pt \\ http://www.ifpr.edu.br
}

\begin{abstract}
The technological advances in Unmanned Aerial Vehicles (UAV) related to energy power structure inspection are gaining visibility in the past decade, due to the advantages of this technique compared with traditional inspection methods. In the particular case of power pylon structure and components, autonomous UAV inspection architectures are able to increase the efficacy and security of these tasks. This kind of application presents technical challenges that must be faced to build real-world solutions, especially the precise positioning and path following for the UAV during a mission. This paper aims to evaluate a novel architecture applied to a power line pylon inspection process, based on the machine learning techniques to process and identify the signal obtained from a UAV-embedded planar Light Detection and Ranging - LiDAR sensor. A simulated environment built on the GAZEBO software presents a first evaluation of the architecture. The results show an positive detection accuracy level superior to $97 \%$ using the vertical scan data and $70 \%$ using the horizontal scan data. This accuracy level indicates that the proposed architecture is proper for the development of positioning algorithms based on the LiDAR scan data of a power pylon.
\end{abstract}

Keywords: UAV LiDAR pylon detection - Detailed electric pylon inspection $\cdot$ Machine learning pylon detection 


\section{Introduction}

Power line pylon inspection is a regular task performed by energy enterprises to ensure energy infrastructure systems' operational security. Power line structures are robust constructions, but deterioration on their components demands preventive maintenance to keep the quality service in power distribution. The detailed pylon inspection is commonly executed by technicians that reach the base of the energy pylon on foot and climb it to observe the details of its components and structure while looking for defects that could compromise the system. These tasks are hard to execute and risky because it is usually carried out with the energy structure in regular operation.

The manufacture advances of small unmanned aircraft, specifically the multirotor types, allows the proposition of new inspection techniques, including the pylon detailed inspections based on this kind of vehicle embedded with regular and thermal cameras. Unmanned Aerial Vehicles (UAV) started being used on this kind of inspection in the last two decades, driven by the offer of cheap and robust small aircraft on the global market. For detailed structure inspection, multi-rotor aircraft presents some advantages, like static flight capability, vertical landing, precise position reaching, and 3D path following, among others. This kind of operation is commonly made with the aircraft remotely piloted by a human operator. To achieve the detailed images properly, the aircraft must fly near the pylon while performing a displacement around it, avoiding obstacles, and keeping a security distance from the structure and components. It is a hard process based on the pilot skills.

Recent research proposes the use of autonomous multi-rotor aircraft to inspect the power line structure. The use of autonomous aircraft for detailed pylon inspections offers some challenges for the proper operation, like precise positioning and path following, effective obstacle detection and collision avoidance, robust flight control and path planning for the operation, component defect identification, among others. Autonomous flight demands a high accuracy position system to be executed properly. The most common approach to providing precise position data to UAV flight currently uses Differential Global Navigation Satellite System (DGNSS). This technique is based on using the difference of satellite signal received between two reception antennas, the first one is placed in a static reference point, and the second one is placed on-board the aircraft, to calculate a high accuracy position output data for the mobile module. The global market presents a considerable number of small DGNSS hardware proper to be used in small aircraft. The technique provides $1.0-\mathrm{cm}$ level horizontal position accuracy but is sensitive to the environment and operational conditions. To face this problem, the proposition of additional position algorithms for UAV precise flight is presented in the literature, mostly based on computer vision systems.

The precise positioning of the aircraft is essential to provide information to the flight controller to assure flight security in a detailed inspection process. In the specific case of the power line structure inspection, some specific challenges are presented, like: 
- Energy power pylons are complex structure, composed of thin metallic components, hard to detect by conventional sensors;

- High voltage energy that flows within the cables generates electromagnetic interference in the navigation sensors of the aircraft during the inspection operation;

- Presence of trees, civil constructions, and other similar objects around the pylons and transmission corridors makes it hard to identify the correct position of the UAV based on intelligent sensor data processing;

- The need for the aircraft flying close to the tower to obtain adequate images and data for analysis brings high risky of collision, a serious problem to this kind of application;

- Maintaining the aircraft's position and orientation for the adequate acquisition of images, due to the presence of gusts of wind and the uncertainty present in the common positioning sensors, is a very demanding task for the autonomous control system;

- DGNSS systems are sensitive to environmental characteristics, like coverage of the receptor antenna, proper satellite signal reception, and data link WiFi reception.

Considering these demands, the proposition of UAV positioning systems based on intelligent computational techniques is an interesting field of application-oriented research. The main objective of this kind of technique is to assure the aircraft control system keep the correct position and orientation, in addition to the trajectory following during the flight. The present work proposes an Artificial Intelligence-based architecture specifically designed for power pylon detection, focused on developing a LiDAR positioning system for autonomous detailed inspection Tasks. It is based on the processing of planar Light Detection And Range (LiDAR) sensors data embedded on the aircraft. This paper presents an initial evaluation of an architecture to detect a pylon in the UAV flight area; it also identifies its direction using only the embedded LiDAR scan data. The simulations were run in the virtual robotic environment GAZEBO, providing an overview of the proposed technique's performance.

The remaining of the paper is organized as follows: After this introductory section, Sect. 2 discusses the related work and highlights this work's contributions. Section 3 presents the general problem description is presented and describes the proposed architecture. Section 4 presents the experimental setup and simulation results, and Sect. 5 discusses the obtained results. Finally, Sect. 6 presents the conclusions and points out future works directions.

\section{Related Works}

The common technique to provide position data to UAV outdoor flight is the use of DGNSS modules. This technique computes the phase difference between two GNSS antennas to increase the accuracy of the positioning data output. Nowadays, a considerable number of small-size DGNSS hardware is commercially available, proper for UAV applications. The most used commercial flight 
controllers can work with these GNSS modules data to provide high accuracy positions to the aircraft, allowing autonomous mission programming. This technique is one of the most common solutions to provide precise horizontal positioning to UAV but demands good operational conditions to work properly. Some environment conditions, like the presence of obstacles, antenna shadowing, magnetic field interference, and cloudy weather conditions, may affect the system accuracy significantly [19], justifying the development of complementary positioning solutions to integrate the UAV control system.

A common approach to propose UAV position and navigation data is using computer vision algorithms that collect images based on regular or stereoscopic cameras embedded on the UAV. Some review papers were published about this subject, presenting the main applications and challenges about the practical use of vision-based UAV control algorithms. This includes the influence of outdoor light variation, difficult of identifying object clues in the images to provide data information for the vision algorithms, and the high variability of the flight sites composition and objects, making it hard to define a general image algorithm processing for all kind of application $[1,10,12]$.

Considering the specific area of energy power structure autonomous inspection, two kinds of computer vision algorithm approaches are found in the literature: (a) the power line following applications, used for a long-range and long-distance visual inspection, and (b) the pylon detection and localization for small distance detailed inspection operation.

The line following applications has the main objective of identifying the position of the UAV related to the energy power cables and transmission structure during its displacement along the lines when the aircraft capture images of the energy cables and components for an overview of the structural conditions. In this situation, the algorithm must keep the UAV navigation in the correct path and distance from the structures during the aircraft displacement $(10.0 \mathrm{~m}$ to $50.0 \mathrm{~m}$ commonly), using the images captured to provide information to the distance and orientation estimators, feeding the flight controller. Most proposals use image processing techniques to extract the power line from an image and calculate the position and orientation of the UAV related to it $[2,6,9,11,13,14,18]$.

Another approach uses pylon detection and identification on images to provide a direction to the UAV displacement. In these cases, the vision algorithms extract the pylon features from the image and calculate its position, feeding the flight control hardware to pursuit the "target" and displace it to the next pylon. An example of this approach is presented in the work [8].

Some works presented in the literature apply vision algorithms to provide a high accuracy position data to the UAV related to the power pylon when it executes the flight in small distances $(2.0 \mathrm{~m}$ to $6.0 \mathrm{~m}$ commonly) to capture detailed images of the structure components.

A pylon distance estimation algorithm based on monocular image processing is presented in [3]. It uses the UAV displacement based on the GPS and the aircraft's IMU (Inertial Measurement Unity) data to calculate the pylon position using the image match points for two consecutive images. A position measure- 
ment error lower than $1.0 \mathrm{~m}$ has been reported as a result (for the best samples) of a $10.0 \mathrm{~m}$ distance flight in real-world experiments, using a pylon model.

Another similar work proposes a "Point-Line-Based SLAM" technique based on a monocular camera to calculate the center of the pylon. A 3D point cloud is estimated from the monocular images at each algorithm interaction, providing the capability to calculate the distance from the UAV and the pylon. The results present an average position error of $0.72 \mathrm{~m}$ [5].

Using LiDAR sensors to provide distance data for energy power inspection is a good approach to solve problems related to the inspection tasks. The main application of this kind of sensor in UAV energy power inspection is focused on the mapping of the structures using the LiDAR point cloud data to reconstruct the real conditions of the transmission lines $[4,9,16,17]$.

Another approach that proposes a distance estimation of a pylon for a detailed inspection application, based on a planar LiDAR sensor, is presented. In the work, a planar LiDAR carried onboard by a multi-rotor aircraft collects horizontal data from the pylon structure and uses such information to calculate the geometric centroid of the pylon. One issue of this technique is that it demands the aircraft to keep the alignment to the pylon to obtain proper data to feed the position calculation. Also, the inclination of the measurement plane, due to the movement of the aircraft, has a significant influence on the position error calculated by the algorithm, as described in [15].

This brief state-of-art review shows that intelligent computing algorithms based on a planar LiDAR sensor data can provide a significant contribution to this specific area of application, especially when they are employed to identify the pylon position/orientation and to calculate its distance from a UAV flying close to it. The work described in the present paper is a first evaluation of the application of machine learning algorithms to face the mentioned challenges.

\section{Problem Description}

The power pylon detailed inspection demands the UAV displacement around its structure. In this operation, the technician goal is to achieve a close-up image of the pylon components to verify their integrity. This operation requires that the aircraft stays hovering close to the targets points, typically within $4.0 \mathrm{~m}$. The robustness of the flight control depends on the correct evaluation of the UAV position, in this case, with high accuracy. Although a centimeter-level accuracy obtained by a DGNSS system is suitable for this operation, the malfunction of this system demands the proposal of additional positioning systems to assure the security of the flight, as explained earlier.

This work main goal is, as described below, to run an initial evaluation of a positioning architecture based on an Artificial Intelligence algorithm to detect an electric power pylon close to a UAV in short distances, in order to assist the detailed pylon inspection tasks. Such an architecture is based on two planar LiDAR sensors to scan, respectively, the horizontal and vertical planes. Each 
sensor scans provide a planar point signature data for intelligent algorithms, to identify correctly the pylon within the flight area and to indicate its structure orientation regarding to the UAV pose.

This research was based on the expertise of the local energy company technicians, which provided essential pieces of information regarding the real-world UAV inspection process. The local company's technicians perform the pylon detailed inspection using a commercial remotely piloted aircraft that captures images from the structure for a offboard post-processing evaluation.

The proposal of UAV-based autonomous pylon inspection architecture must assure the capability of reproducing the human-piloted behavior, keeping the flight security during the operation. An important demand regarding this is to identify the presence of the pylon in front of the UAV and keep a secure distance from the structure, to prevent a possible collision. The use of a LiDAR sensor embedded on the UAV is a possible way to estimate the distance between the aircraft and the pylon in close distances. To work properly the flight control system must receive reliable information that the structure detected by the LiDAR is the pylon, once there are several other possible objects in the flight area that could be detected, generating a false distance estimation. Considering this, a pylon detection and identification algorithm must be provided.

To face these challenges, this work proposes an architecture based on two planar LiDAR sensors embedded on the UAV, to scan both the horizontal and the vertical planes and feed AI algorithms. To allow the correct operation of the algorithms, a predefined programmed behavior for the UAV was proposed. The Fig. 1 shows the representation of the UAV proposed behavior for a inspection process.

Two different AI algorithms have been evaluated to compare the pylon detection performance in the simulated environment: a Neural Network (NN), and a Support Vector Machine (SVM). Both of these algorithms were chosen for being well documented in the literature and widely applied in classification problems, and, therefore, they are a good baseline for this evaluation. A deep Feed Forward Network (FFN) was designed in Python (v3.8.5) with the aid of the Keras (v2.4.1) framework. The NN was build with three hidden dense layers, with the first two being composed by 200 neurons each and a third layer composed by 5 neurons. Each layer uses the RelU activation function, and the output's layer generates the final result using the sigmoid activation function. The network was trained for 50 epochs and with batch size of 32 . Accuracy was chosen as the evaluation metric, and the loss function used was binary crossentropy. Also, to mitigate overfitting, the cross-validation technique was used, with the training data being split in 10 sets. The network training was executed as an supervised model, were each sample was tagged with the "pylon" or "not-pylon" boolean parameter.

The Support Vector Machine (SVM) algorithm has been designed using Python (v3.8.5) and the scikit-learn library. Two kernels for parameter estimation have been used: the polynomial and the radial basis function (RBF). Similar to the NN algorithm, the SVM algorithm training has been executed 


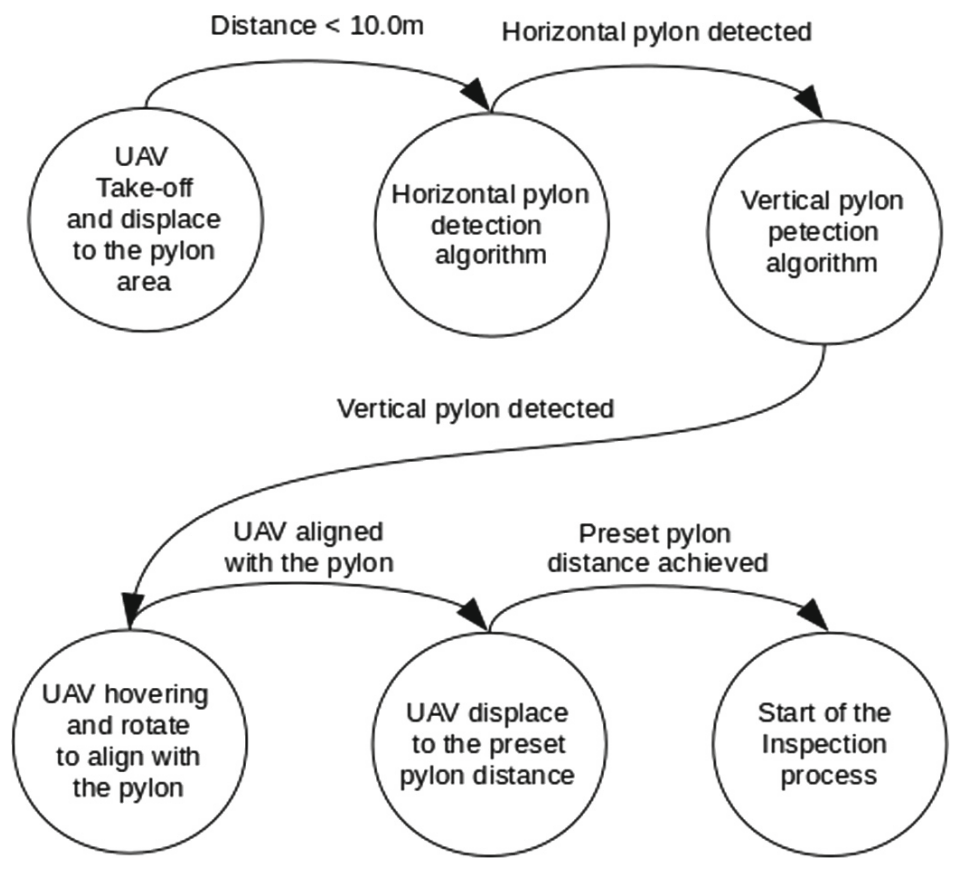

Fig. 1. State diagram showing the proposed UAV behavior for a inspection process.

in the supervised model using the binary parameter to classify the samples as "pylon" or "not-pylon".

\section{Experimental Setup}

To validate the architecture, several simulated scenarios containing an energy pylon and/or other objects were developed. In each of them, samples of the LiDAR were collected and fed to both an SVM and a NN algorithm. They were evaluated using the following metrics: overall performance, performance on each scenario, and execution time. The overall performance was evaluated using mainly accuracy as a metric. The performance on each scenario was measured to find the effects different scenarios may have on the detection ratio. And at last, a time comparison between the algorithms will be shown, as time is a crucial metric for applications running in embedded systems.

The validation experiments have been executed on a virtual environment built within the Gazebo Multi-Robot Simulator, version 11.3.0 running on a Ubuntu 20.04 Operating System with ROS Noetic Ninjemys. The Gazebo simulation software and the AI algorithms run on a PC equipped with an Intel i5 10400 processor, 16 Gb RAM and a GPU NVIDIA GeForce 1660 Super with 6 Gb RAM. Two standard planar LiDAR sensor models have been used to scan the horizontal and the vertical planes. These sensors have a 40.0-m range, sampling rate of 5 samples per-second, and angle steps of $0.5^{\circ}$. Also, for the horizontal plane, the LiDAR sensor scans $360.0^{\circ}$ around the UAV; for the vertical plane, the sensor scans $90.0^{\circ}$ on the front side of the UAV. 
Eight distinct scenarios were built to allow the data collection for the training and evaluation of the horizontal AI algorithms, as shown in Fig. 2.

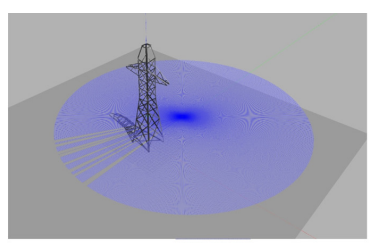

a)

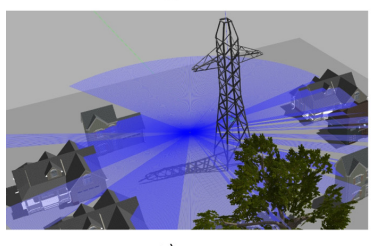

e)

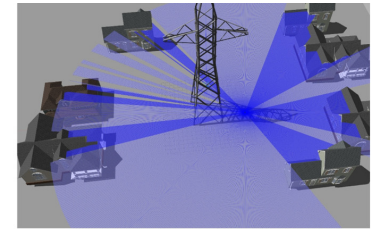

b)

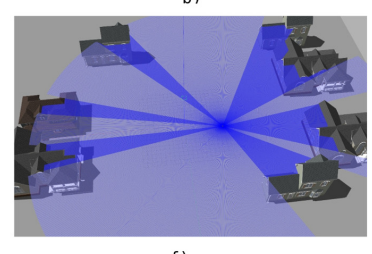

f)

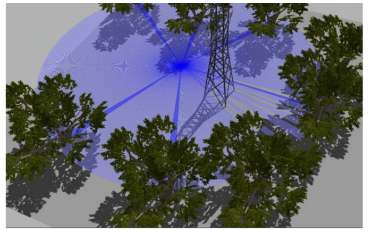

c)

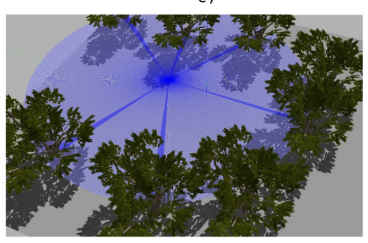

g)
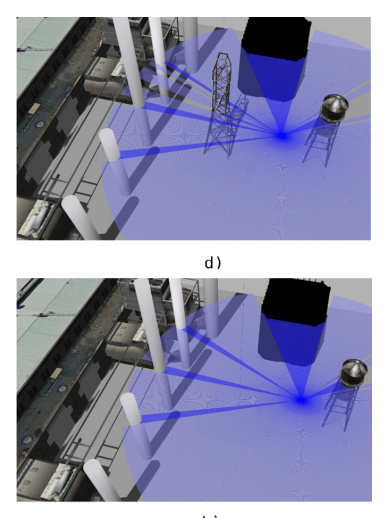

Fig. 2. Images of the experiment scenarios. a) Only a pylon in the field. b) Several buildings and one pylon. c) Several trees and one pylon in the field. d) A complex building structure, a water tank and one pylon. e) Several buildings, one pylon and one tree. f) Several buildings; no pylon. g) Several trees in the field close from each other; no pylon. h) A complex building structure, a water tank; no pylon.

A 3D STL pylon model from GRABCAD [7] website was imported to the GAZEBO. To execute the data collection for the horizontal LiDAR, the sensor was randomly displaced around the pylon on a range from $2.0 \mathrm{~m}$ to $10.0 \mathrm{~m}$ in different heights between $2.0 \mathrm{~m}$ and $20.0 \mathrm{~m}$. The pylon height was set to $30.0 \mathrm{~m}$. For all the experiments a practical approach was considered in the sensor horizontal stabilization. It was defined that the LiDAR sensor was attached to a stabilized gimbal that keeps the sensor always scanning in a horizontal or vertical plane. A random horizontal alignment noise with 1.0-degree range was added to the sensor position to simulate the stabilization error present on the gimbal mechanism. Figure 3 shows an example of the horizontal point map LiDAR detection on a simulation in a complex environment.

For each position, the sensor captures a 360-degree planar point data and store it into a vector. All samples for each scenario were stored and pos-processed, indicating the presence or not presence of the pylon in the scenario. The training set was composed of $80 \%$ of the collected samples and the test set was composed of $20 \%$ of the collected samples.

Table 1 presents the results of the SVM algorithm versus the NN algorithm for the horizontal LiDAR data experiment.

For the vertical data collection, the LiDAR sensor was configured in the same conditions as the horizontal sensor, however, it scans a 90.0-degree range. A total of 17500 samples have been captured and used as the training and test data. The main difference for this data collection is that the sensor was keep pointed to the pylon in the scenario with a pylon present to assure that the collected samples contain the detection of the pylon segment. These samples have been tagged as "pylon" to feed the training set. In the other scenarios, the sensor was randomly placed in the space inside a $10.0-\mathrm{m}$ radius from the center of the scene, and also 


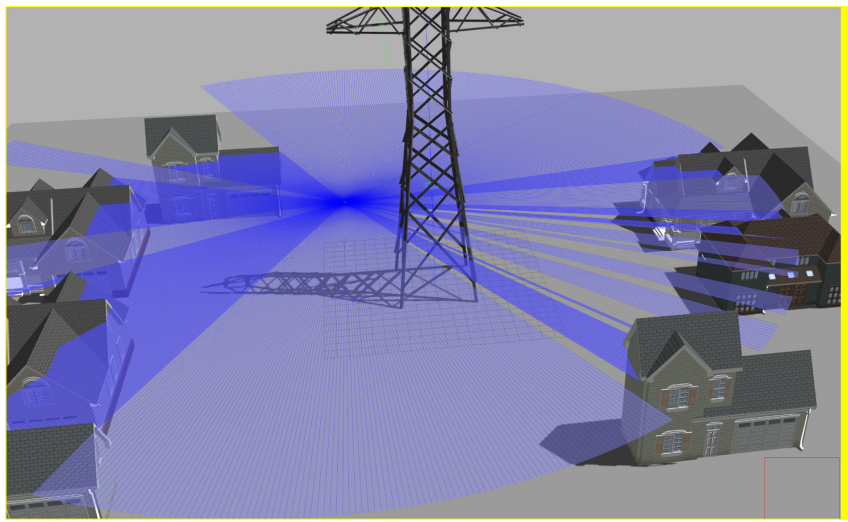

a)

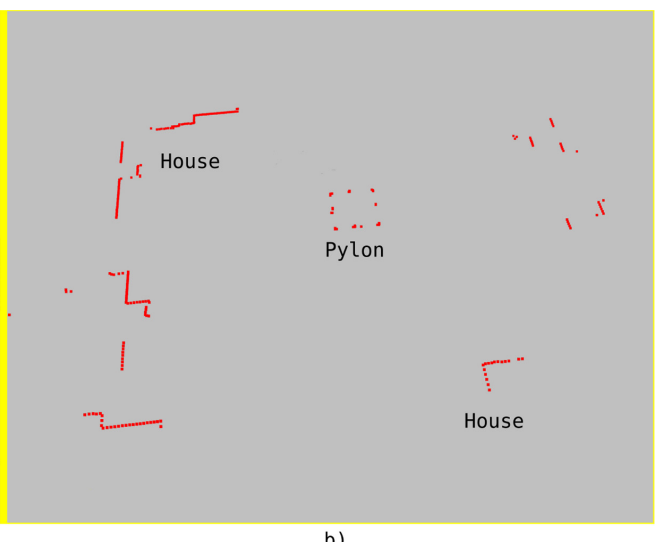

Fig. 3. a) Simulation scene on GAZEBO showing the pylon, objects in the area and the sensor scan. b) The point map of the horizontal LiDAR detection.

Table 1. SVM $\times$ NN comparing in horizontal LiDAR data set

\begin{tabular}{|c|c|c|c|c|c|c|c|}
\hline \multicolumn{4}{|c|}{$\begin{array}{c}\text { Horizontal SVM } \\
\text { Training Set Evaluation }\end{array}$} & \multicolumn{4}{|c|}{$\begin{array}{c}\text { Horizontal NN } \\
\text { Training Set Evaluation }\end{array}$} \\
\hline & \multicolumn{3}{|c|}{ Predicted Result } & & \multicolumn{3}{|c|}{ Predicted Result } \\
\hline \multirow{3}{*}{ Actual Result } & & True & False & \multirow{3}{*}{ Actual Result } & & True & False \\
\hline & True & 1830 & 166 & & True & 1562 & 434 \\
\hline & False & 179 & 825 & & False & 416 & 588 \\
\hline \multicolumn{8}{|c|}{ Total of Samples: 3000} \\
\hline $\begin{array}{c}\text { True } \\
\text { Readings }\end{array}$ & 2655 & $\begin{array}{l}\text { Percentual } \\
\text { Accuracy }\end{array}$ & $88.50 \%$ & $\begin{array}{c}\text { True } \\
\text { Readings }\end{array}$ & 2150 & $\begin{array}{l}\text { Percentual } \\
\text { Accuracy }\end{array}$ & $71.67 \%$ \\
\hline
\end{tabular}

randomly directed to collect data from the structures and elements present in the environment. These samples were tagged as "no pylon" to feed the training set. From these datasets, $80 \%$ of the samples were used as the training dataset and $20 \%$ for the test dataset. Table 2 presents the results of the SVM and NN algorithms for the vertical LiDAR data experiment.

Table 2. SVM $\times$ NN comparing the vertical LiDAR data set

\begin{tabular}{|c|c|c|c|c|c|c|c|}
\hline \multicolumn{4}{|c|}{$\begin{array}{c}\text { Vertical SVM } \\
\text { Training Set Evaluation }\end{array}$} & \multicolumn{4}{|c|}{$\begin{array}{c}\text { Vertical NN } \\
\text { Training Set Evaluation }\end{array}$} \\
\hline & \multicolumn{3}{|c|}{ Predicted Result } & & \multicolumn{3}{|c|}{ Predicted Result } \\
\hline \multirow{3}{*}{ Actual Result } & & True & False & \multirow{3}{*}{ Actual Result } & & True & False \\
\hline & True & 1991 & 24 & & True & 1997 & 38 \\
\hline & False & 47 & 1438 & & False & 60 & 1425 \\
\hline \multicolumn{8}{|c|}{ Total of Samples: 3500} \\
\hline $\begin{array}{c}\text { True } \\
\text { Readings }\end{array}$ & 3429 & $\begin{array}{l}\text { Percentual } \\
\text { Accuracy }\end{array}$ & $97.97 \%$ & $\begin{array}{c}\text { True } \\
\text { Readings }\end{array}$ & 3420 & $\begin{array}{c}\text { Percentual } \\
\text { Accuracy }\end{array}$ & $97.20 \%$ \\
\hline
\end{tabular}


The performance of the NN and SVM algorithms for each kind of scenario is shown in the Fig. 4.
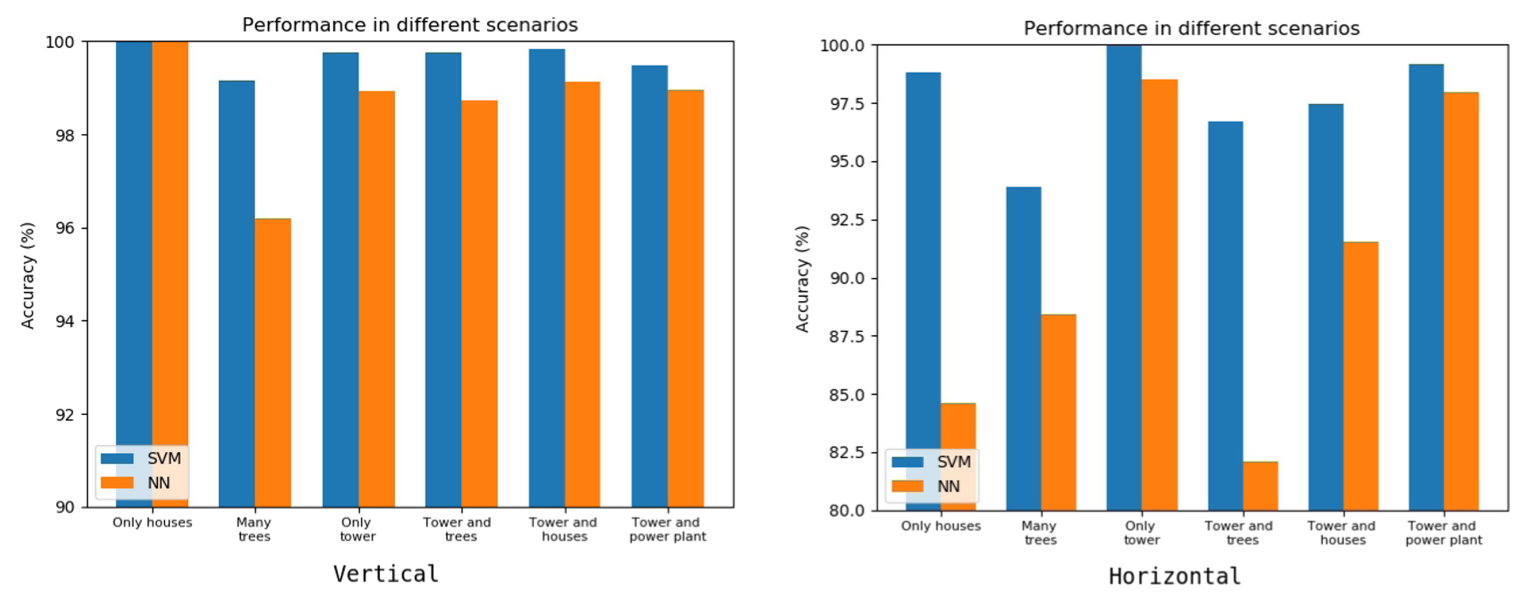

Fig. 4. Accuracy performance of the SVM algorithm versus NN algorithm for the different experimentation scenarios. SVMs had a better overall performance in every scenario.

\subsection{SVM $\times$ NN Time Performance}

The time performance of the AI algorithm is an important parameter to the practical application of the architecture. To evaluate this performance, the same number of predictions were performed for each algorithm for both the horizontal and vertical LiDAR datasets to calculate the average time for a single prediction. As the real UAV will run using previously trained models, the training times will not have influence in the UAV performance, and thus only the prediction times were measured. The results are presented in Fig. 5.

\section{Discussion}

Considering the constructive characteristics of the energy pylon, which is composed of thin metallic segments, the planar LiDAR data collected offers few points to the AI algorithms processing. This is an important parameter to be considered in training phase. The LiDAR sensors can capture points not only from the face of the pylon but also from the back of the structure, and thus, the point map is particularly different from other objects or buildings commonly found in the surroundings of an energy transmission line area. In spite of the LiDAR sensors being used for distance measurements mainly, the point signature generated in a pylon scanning in comparison with other objects. The results obtained in our simulated experiments show the likelihood that the proposed approach can provide suitable information to train an AI-based classification algorithm for real-world applications.

As described earlier, the proposed architecture provides a way for the UAV to detect a pylon present in the flight area and to rotate it to find pylon direction. 

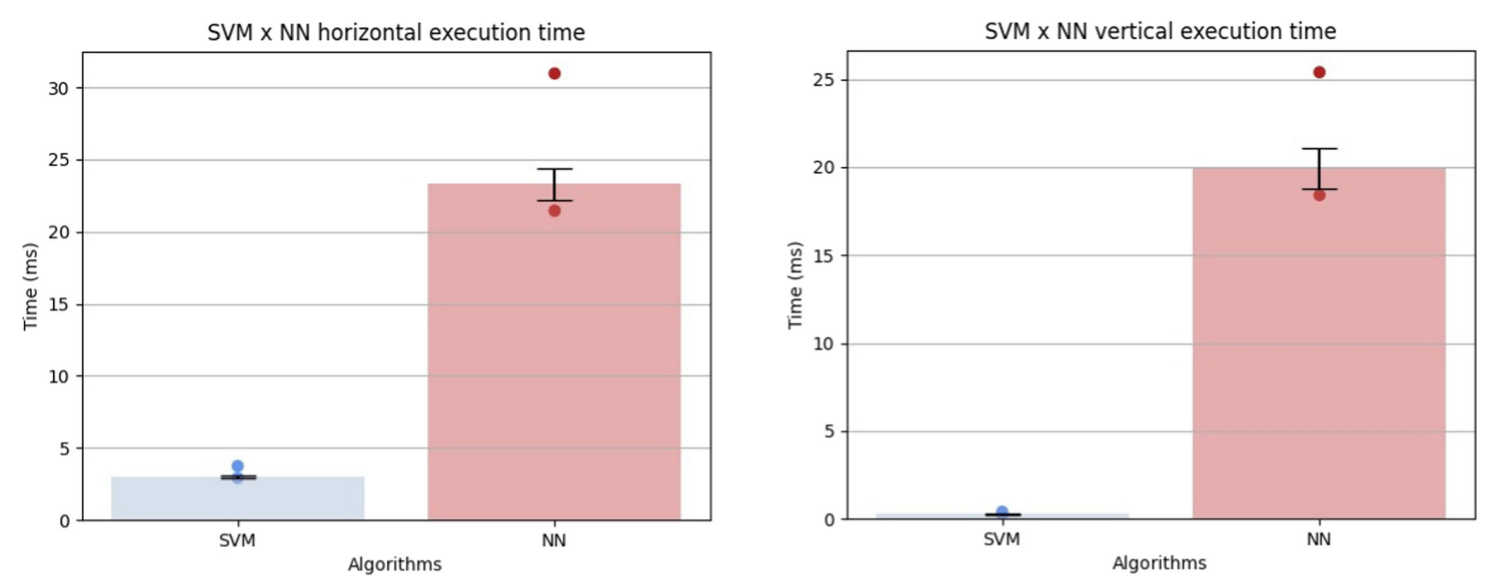

Fig. 5. Time performance of the SVM algorithm versus NN algorithm for the horizontal and vertical LiDAR data. The results show that the prediction times using the SVM algorithm were much faster than their NN counterpart.

This allows the UAV to keep its front-side pointed to the pylon face during all the inspection process. Moreover, assuring that the pylon exists in the environment and the UAV is pointed to it, a more reliable distance measurement system based on the LiDAR data readings could be implemented. The proposal of new distance measurement algorithms is planned for the next steps of this research.

Comparing the two AI algorithms fed with the horizontal data has shown an accuracy of $88.50 \%$ for the SVM against $71.67 \%$ for the NN. For this experiment's scenarios, the results indicate that the SVM algorithm is better than the NN considering only the accuracy metric. Also the SVM algorithm average processing time for each prediction is about significantly smaller than the NN processing time. It is important to remember that, to be employed in realworld applications, this architecture intends to be deployed on UAV's onboard embedded computing system, which may present limited hardware resources and performance. Therefore, the choice of which algorithm should compose the detection architecture must be considered carefully.

The vertical LiDAR data have shown a similar performance between the SVM and NN algorithms, with an accuracy of $97.97 \%$ and $97.20 \%$, respectively. The vertical scan of the pylon allows detecting more segments of the structure, offering a detailed signal signature as input to the AI algorithms.

Different kind of scenarios presents different performances for the algorithms, as expected. It is possible to observe that in the specific case where a significant number of trees are present in the area, the accuracy of the algorithms has a significant reduction. Besides that, in a real-world application, the algorithm will operate in a distance not greater than 10.0-meter from the pylon, where the presence of trees is not common. Also is possible to observe in the Fig. 4 that the SVM algorithm provides better performance for all the evaluations.

Comparing the processing time performance, the SVM processing is five times longer than the NN processing. This impacts the detection algorithm execution frequency, which, in turn, imposes a speed constraint to the aircraft displacement speed. 


\section{Conclusions and Future Works}

This work goal was to evaluate the use of AI-based algorithms to compose an architecture capable of detecting a power pylon structure in the flight area of a UAV used for detailed inspection applications. Such an architecture must offer a reliable response about the direction of the pylon, by detecting its structure using a planar LiDAR sensor aligned to the vertical plane of the aircraft.

Although a planar LiDAR scan data may provide less information to feed an AI-based detection algorithm (in comparison with, e.g., an image-based dataset), the results obtained in the simulated environment present good potential for a real-world architecture implementation. The obtained accuracy ranged from $70 \%$ to $99 \%$, depending on the arrangement and applied AI algorithm. It is possible to state intuitively that the processing time of LiDAR samples is considerably shorter than an image. Therefore, the proposed pylon detecting architecture based on LiDAR sensors data can be deployed to the UAV's onboard embedded computing system.

The algorithm comparison has shown that the SVM-based architecture provides better results than the NN-based architecture for this kind of application. These results indicate that the construction of real-world application architecture will probably present better results by using this kind of algorithm. Also, the SVM processing time was significantly smaller than the NN one, which probably will be reproduced in a real-world situation.

This work pioneers in proposing an approach based on LiDAR data and IA-based classification algorithms to detect energy power pylons to the best of our knowledge. Two popular AI algorithms have been evaluated for this task. Although computer vision algorithms are probably the most common approach to detecting a pylon in the captured images, those algorithms are used to calculate the distance between the pylon and the UAV or provide visual odometry. However, the amount of data provided by a single image is huge compared to a planar LiDAR data sample, demanding not only a higher amount of processing and memory resources but also an additional financial cost to build the embedded computing system. Thus, it is possible to say that using an architecture such as the one proposed in this work has a good potential to be implemented in real-world applications. It demands less processing time while providing reliable information about the presence of a pylon in the flight area and its direction related to the UAV pose. This work is the first step towards implementing a position algorithm based on the measurements of the pylon structure captured by the LiDAR sensors.

The results presented in this work represent a first evaluation of the IA-based algorithms to detect a electric pylon based on LiDAR point scans. Future work will evaluate the real-world performance of the proposed architecture using data collected from a scale-size pylon and LiDAR sensors carried onboard of a smallsize quad-rotor aircraft. A distance evaluation algorithm based on the LiDAR readings is also foreseen as future work. Such a system intends to provide relative positioning data to the UAV flight controller. 
Acknowledgements. This work has been supported by FCT - Fundação para a Ciência e Tecnologia within the Project Scope: UIDB/05757/2020. This work has also been supported by Fundação Araucária (grant 34/2019), and by CAPES and UTFPR through stundent scholarships.

\section{References}

1. Al-Kaff, A., Martín, D., García, F., de la Escalera, A., María Armingol, J.: Survey of computer vision algorithms and applications for unmanned aerial vehicles. Expert Syst. Appl. 92, 447-463 (2018)

2. Araar, O., Aouf, N.: Visual servoing of a Quadrotor UAV for autonomous power lines inspection. In: 2014 22nd Mediterranean Conference on Control and Automation, MED 2014 (June), pp. 1418-1424 (2014). https://doi.org/10.1109/MED. 2014.6961575

3. Araar, O., Aouf, N., Dietz, J.L.V.: Power pylon detection and monocular depth estimation from inspection UAVs. Ind. Robot. 42(3), 200-213 (2015). https://doi. org/10.1108/IR-11-2014-0419

4. Azevedo, F.: LiDAR-based real-time detection and modeling of power lines for unmanned aerial vehicles. Sensors (Switzerland) 19(8), 1-28 (2019). https://doi. org $/ 10.3390 / \mathrm{s} 19081812$

5. Bian, J., Hui, X., Zhao, X., Tan, M.: A point-line-based SLAM framework for UAV close proximity transmission tower inspection. In: 2018 IEEE International Conference on Robotics and Biomimetics, ROBIO 2018, pp. 1016-1021 (2019). https://doi.org/10.1109/ROBIO.2018.8664716

6. Cerón, A., Mondragón, I., Prieto, F.: Onboard visual-based navigation system for power line following with UAV. Int. J. Adv. Rob. Syst. 15(2), 1-12 (2018). https:// doi.org/10.1177/1729881418763452

7. GRABCAD: GrabCAD (2021). https://grabcad.com/

8. Hui, X., Bian, J., Yu, Y., Zhao, X., Tan, M.: A novel autonomous navigation approach for UAV power line inspection. In: 2017 IEEE International Conference on Robotics and Biomimetics, ROBIO 2017, 1-6 January 2018 (2018). https://doi. org/10.1109/ROBIO.2017.8324488

9. Li, X., Guo, Y.: Application of LiDAR technology in power line inspection. IOP Conf. Ser.: Mater. Sci. Eng. 382(5), 1-5 (2018). https://doi.org/10.1088/1757899X/382/5/052025

10. Máthé, K., Buşoniu, L.: Vision and control for UAVs: a survey of general methods and of inexpensive platforms for infrastructure inspection. Sensors (Switzerland) 15(7), 14887-14916 (2015)

11. Menéndez, O., Pérez, M., Cheein, F.A.: Visual-based positioning of aerial maintenance platforms on overhead transmission lines. Appl. Sci. (Switz.) 9(1) (2019). https://doi.org/10.3390/app9010165

12. Nguyen, V.N., Jenssen, R., Roverso, D.: Automatic autonomous vision-based power line inspection: a review of current status and the potential role of deep learning. Int. J. Electr. Power Energy Syst. 99(January), 107-120 (2018)

13. Shuai, C., Wang, H., Zhang, G., Kou, Z., Zhang, W.: Power lines extraction and distance measurement from binocular aerial images for power lines inspection using UAV. In: Proceedings - 9th International Conference on Intelligent HumanMachine Systems and Cybernetics, IHMSC 2017, vol. 2, 69-74 (2017). https://doi. org/10.1109/IHMSC.2017.131 
14. Tian, F., Wang, Y., Zhu, L.: Power line recognition and tracking method for UAVs inspection. In: 2015 IEEE International Conference on Information and Automation, ICIA 2015 - In conjunction with 2015 IEEE International Conference on Automation and Logistics (August), pp. 2136-2141 (2015). https://doi.org/10. 1109/ICInfA.2015.7279641

15. Viña, C., Morin, P.: Micro air vehicle local pose estimation with a two-dimensional laser scanner: a case study for electric tower inspection. Int. J. Micro Air Veh. 10(2), 127-156 (2018). https://doi.org/10.1177/1756829317745316

16. Wu, J., Fei, W., Li, Q.: An integrated measure and location method based on airborne 2D laser scanning sensor for UAV's power line inspection. In: Proceedings - 2013 5th Conference on Measuring Technology and Mechatronics Automation, ICMTMA 2013, pp. 213-217 (2013). https://doi.org/10.1109/ICMTMA.2013.58

17. Zhang, W., et al.: The application research of UAV-based LiDAR system for power line inspection. In: Proceedings of the 2nd International Conference on Computer Engineering, Information Science \& Application Technology (ICCIA 2017), vol. 74, pp. 962-966 (2017). https://doi.org/10.2991/iccia-17.2017.174

18. Zhao, X., Tan, M., Hui, X., Bian, J.: Deep-learning-based autonomous navigation approach for UAV transmission line inspection. In: Proceedings - 2018 10th International Conference on Advanced Computational Intelligence, ICACI 2018, pp. 455-460 (2018). https://doi.org/10.1109/ICACI.2018.8377502

19. Zimmermann, F., Eling, C., Klingbeil, L., Kuhlmann, H.: Precise positioning of UAVs - dealing with challenging RTK-GPS measurement conditions during automated UAV flights. ISPRS Ann. Photogramm. Remote Sens. Spat. Inf. Sci. 4(2W3), 95-102 (2017). https://doi.org/10.5194/isprs-annals-IV-2-W3-95-2017 